\title{
A Class of Nonlinear Stochastic Volatility Models
}

\author{
Jun Yu, Zhenlin Yang \\ Singapore Management University
}

\begin{abstract}
This paper proposes a class of nonlinear stochastic volatility (SV) models based on the Box-Cox transformation. The proposed class encompasses many parametric SV models that have appeared in the literature, including the well known lognormal SV model, and has an advantage in the ease with which different specifications on SV can be tested. In addition, the functional form of transformation which induces marginal normality of volatility is obtained as a byproduct of this general way of modeling SV. Efficient method of moments is used to estimate the model. Empirical results reveal that the lognormal SV model is rejected.
\end{abstract}

\section{Keywords: Box-Cox Transform; EMM; GARCH}

\section{Introduction}

Modeling the volatility of financial time series via stochastic volatility (SV) models has received a great deal of attention both in the theoretical finance and in the empirical finance literature. Prices of options based on SV models are shown to be more accurate than those based on the Black-Scholes model (see, for example, Melino and Turnbull (1990)). Moreover, the SV model is proved to be a serious competitor to GARCH-type models for explaining time varying volatility. Empirical successes of the lognormal SV model relative to GARCH are documented in $\mathrm{Kim}$, et al. (1998) in terms of in-sample fitting, and in $\mathrm{Yu}$ (2002) in terms of out-of-sample forecasting.

In the option pricing literature, the SV model is often formulated in terms of stochastic differential equations. For instance, Wiggins (1987), Chesney and Scott (1989), and Scott (1987) specify the following model for the asset price $P(t)$ and the corresponding volatility $\sigma^{2}(t)$,

$$
\begin{gathered}
d P(t) / P(t)=\alpha d t+\sigma(t) d B_{1}(t), \\
d \ln \sigma^{2}(t)=\lambda\left(\xi-\ln \sigma^{2}(t)\right) d t+\gamma d B_{2}(t),
\end{gathered}
$$

where $B_{1}(t)$ and $B_{2}(t)$ are two independent standard Brownian motions.

In the empirical literature, the above continuous time model is often discretized. For example, the EulerMaruyama The approximation, after a location shift and reparameterization, leads to the so-called lognormal SV model given by

$$
\begin{gathered}
X_{t}=\sigma_{t} e_{t}, \\
\ln \sigma_{t}^{2}=\mu+\phi\left(\ln \sigma_{t-1}^{2}-\mu\right)+\sigma v_{t},
\end{gathered}
$$

where $X_{t}$ is the continuously compounded return and $e_{t}, v_{t}$ are two uncorrelated sequences of independent and identically distributed (iid) $N(0,1)$ variables. In the literature $\sigma_{t}$ is often represented by $\exp \left(\frac{1}{2} h_{t}\right)$. One implication of its specification is that the marginal distribution of logarithmic volatility is normal. This assumption has very important implications for financial economics and risk management. So far this model as received a great deal of attention in the econometrics literature.

Alternative SV models have appeared in the literature. For example, Stein and Stein (1991) and Johnson and Shanno (1987) assume $\sigma(t)$ follows, respectively, an Ornstein-Uhlenbeck (OU) process and a geometric Brownian motion, while Hull and White (1987) and Heston (1993) assume a geometric Brownian motion and a squareroot process for $\sigma^{2}(t)$. In the discrete time case, various SV models can be regarded as generalizations to corresponding GARCH models. For example, a polynomial $\mathrm{SV}$ model is a generalization of $\operatorname{GARCH}(1,1)$ (Bollerslev (1986)) while a square root polynomial SV model is a generalization of standard deviation $\operatorname{GARCH}(1,1)$. Andersen (1994) introduces a general class of SV models, of which a class of polynomial SV models has been emphasized. This class encompasses many discrete time SV models in the literature.

Despite all these alternative specifications, there is a lack of procedure for selecting appropriate functional form of SV. The specification of the correct SV function, on the other hand, is very important in two respects. First, different functional forms lead to different formulae for option pricing. Misspecification of the SV function can result in incorrect option prices. Second, the marginal distribution of volatility depends upon the functional form of SV.

In this paper, we propose a new class of SV models, namely, the nonlinear SV model. Like the class of Andersen (1994), it includes as special cases many discrete time SV models that have appeared in the literature. It overlaps with but does not encompass the class of Andersen. 
An advantage of our proposed class is the ease with which different specifications on SV can be tested. In fact, the specification test is based on a single parameter. Another advantage of our proposed class is that, as a byproduct of this general way of modeling volatility, one obtains the functional form of transformation which induces marginal normality of volatility. Section 2 presents this class of nonlinear models. In Section 3, we use efficient method of moments (EMM) to estimate the model. In Section 4, the class is fitted to daily observations on an index return.

\section{A Class of Nonlinear SV Models}

The lognormal SV model specifies that the logarithmic volatility follows an $\mathrm{AR}(1)$ process. However, this relationship may not always be warranted by the data. A natural generalization to this relationship is to allow a general (nonlinear) smooth function of volatility to follow an $\operatorname{AR}(1)$ process. That is,

$$
\begin{aligned}
X_{t} & =\sigma_{t} e_{t} \\
h\left(\sigma_{t}^{2}, \delta\right) & =\mu+\phi\left[h\left(\sigma_{t-1}^{2}, \delta\right)-\mu\right]+\sigma v_{t},
\end{aligned}
$$

where $e_{t}$ and $v_{t}$ are two uncorrelated $N(0,1)$ sequences, and $h$ is a smooth function indexed by a parameter $\delta$. A nice choice of this function is the Box-Cox power function (Box and Cox (1964)):

$$
h(t, \delta)= \begin{cases}\left(t^{\delta}-1\right) / \delta, & \text { if } \delta \neq 0, \\ \log t, & \text { if } \delta=0 .\end{cases}
$$

As the function $h$ is specified as a general nonlinear function, we call the model the nonlinear SV model. Several attractive features of this new class of SV models include: i) as we will show below it includes the lognormal SV model and the other "classical" SV models as special cases, ii) it adds great flexibility on the functional form, and iii) it allows a simple test for the lognormal SV specification, i.e., a test of $H_{0}: \delta=0$, and some other "classical" $\mathrm{SV}$ specifications. If we write $h_{t}=h\left(\sigma_{t}^{2}, \delta\right)$, then we can re-write the nonlinear SV models as

$$
\begin{aligned}
X_{t} & =\left[g\left(h_{t}, \delta\right)\right]^{1 / 2} e_{t}, \\
h_{t} & =\mu+\phi\left(h_{t-1}-\mu\right)+\sigma v_{t},
\end{aligned}
$$

where $g\left(h_{t}, \delta\right)$ is the inverse Box-Cox transformation:

$$
g\left(h_{t}, \delta\right)= \begin{cases}\left(1+\delta h_{t}\right)^{1 / \delta}, & \text { if } \delta \neq 0 \\ \exp \left(h_{t}\right), & \text { if } \delta=0\end{cases}
$$

Equivalently we can re-write them in a form of

$$
\begin{gathered}
X_{t}=\sigma_{t} e_{t} \\
\frac{\left(\sigma_{t}^{2}\right)^{\delta}-1}{\delta}=\mu+\phi\left[\frac{\left(\sigma_{t-1}^{2}\right)^{\delta}-1}{\delta}-\mu\right]+\sigma v_{t} .
\end{gathered}
$$

Table 1: Alternative Stochastic Volatility Models and Parameter Relationship

\begin{tabular}{c|c|c|c|c}
\hline & Models & $\delta$ & $\mu$ & $\phi$ \\
\hline $\begin{array}{c}\text { Wiggins } \\
\text { Scott } \\
\text { Kim et al. }\end{array}$ & $\begin{array}{c}\ln \sigma_{t}^{2}=\mu+ \\
\left.\sigma_{t-1}^{2}-\mu\right)+\sigma v_{t}\end{array}$ & 0 & & \\
\hline $\begin{array}{c}\text { Scott } \\
\text { Andersen }\end{array}$ & $\begin{array}{c}\sigma_{t}=\mu+ \\
\left.\sigma_{t-1}-\mu\right)+\sigma v_{t}\end{array}$ & 0.5 & & \\
\hline Heston & $\sigma_{t}=\phi \sigma_{t-1}+\sigma v_{t}$ & 0.5 & 0 & \\
\hline Hull \& White & $\begin{array}{c}\ln \sigma_{t}^{2}=\mu+ \\
\ln \sigma_{t-1}^{2}+\sigma v_{t}\end{array}$ & 0 & & 1 \\
\hline Andersen & $\begin{array}{c}\sigma_{t}^{2}=\mu+ \\
\left(\sigma_{t-1}^{2}-\mu\right)+\sigma v_{t}\end{array}$ & 1 & & \\
\hline Clark & $\ln \sigma_{t}^{2}=\mu+\sigma v_{t}$ & 0 & & 0 \\
\hline $\begin{array}{c}\text { Nonlinear } \\
\text { SV }\end{array}$ & $\begin{array}{c}\left(\left(\sigma_{t}^{2}\right)^{\delta}-1\right) / \delta=\mu+ \\
\left.\phi\left[\left(\sigma_{t-1}^{2}\right)^{\delta}-1\right) / \delta-\mu\right] \\
+\sigma v_{t}\end{array}$ & & & \\
\hline
\end{tabular}

Denote the parameters of interest by $\theta=(\mu, \delta, \phi, \sigma)$.

The idea of our proposed SV models is similar to that in Higgins and Bera (1992) from the linear ARCH model (Engle (1982)) to the nonlinear ARCH (NARCH) model. Obviously, our model provides a stochastic volatility generalization of a nonlinear $\operatorname{GARCH}(1,1)$ model.

It can be seen as $\delta \rightarrow 0,\left(1+\delta h_{t}\right)^{1 /(2 \delta)} \rightarrow \exp \left(0.5 h_{t}\right)$ and $\left(\left(\sigma_{t}^{2}\right)^{\delta}-1\right) / \delta \rightarrow \ln \sigma_{t}^{2}$. Hence the proposed nonlinear SV model includes the lognormal SV model as a special case. If $\delta=1$, the variance equation (0.12) becomes

$$
\sigma_{t}^{2}=\mu^{\prime}+\phi\left(\sigma_{t-1}^{2}-\mu^{\prime}\right)+\sigma v_{t},
$$

where $\mu^{\prime}=\mu+1$. This is a polynomial SV model in Andersen (1994). According to this specification, volatility follows a normal distribution as its marginal distribution. If $\delta=0.5$, the variance equation $(0.12)$ becomes

$$
\sigma_{t}=\mu^{\prime \prime}+\phi\left(\sigma_{t-1}-\mu^{\prime \prime}\right)+0.5 \sigma v_{t},
$$

where $\mu^{\prime \prime}=0.5 \mu+1$. This is a square root polynomial SV model in Andersen (1994) and can be regarded as a discrete time version of the continuous time SV model in Scott (1987) and Stein and Stein (1991). As a result, the marginal distribution of the square root of volatility is Gaussian.

In Table 1 we summarize some well-known stochastic volatility models and show their parameter relations with our model. For the continuous time stochastic volatility models, their Euler discrete time versions are considered. It can be seen that all these models can be obtained from 
our model by placing the appropriate restrictions on the three parameters $\delta, \mu$ and $\phi$. In fact, all the models except ours require $\delta$ to be $0,0.5$, or $1 .^{1}$ For a general $\delta$, our model is different from any of them and $\delta$ provides some idea about the degree of departure from a "classical" parametric SV model.

The Box-Cox transformation has been applied in various areas in finance. One of the most relevant applications to our work may be that by Higgins and Bera (1992) who introduce the nonlinear ARCH model. Another relevant application is Hentschel (1995) who introduces a family of GARCH models by applying the Box-Cox transformation to the conditional standard deviation. A nice feature of our proposed class is that it provides a simple way to test the null hypothesis of polynomial SV specifications, including the lognormal SV specification, against a variety of non-polynomial alternatives. In fact, this specification test is based entirely on a single parameter, $\delta$. Moreover, as a consequence of specification testing, our proposed class provides an effective channel to check the marginal distribution of unobserved volatility. Therefore, our method serves as an alternative approach for studying marginal distribution of daily volatility to the approach based on ultra-high frequency data that has appeared in a recent literature (cf Andersen et al., 2001).

\section{Estimation by EMM}

In this paper we use EMM to estimate the proposed model and to make inferences. EMM is first introduced by Gallant and Tauchen (1996) and has now found many successful applications in economics and finance; see Gallant and Tauchen (2001a) for a brief review of the literature. It is closely related to GMM of Hansen (1982). An important difference between them is that while GMM relies on an ad hoc chosen set of moment conditions, EMM is based on a judiciously chosen set of moment conditions. The moment conditions EMM employs is the expectation of the score of an auxiliary model which is often referred to as the score generator.

Let the SV model be the structural model. The conditional density of the structural model is defined by

$$
p_{t}\left(x_{t} \mid y_{t}, \theta\right)
$$

where $y_{t}$ is a vector of lagged $x_{t}$, the true value of $\theta$ is $\theta_{0}$ and $\theta_{0} \in \Theta \subset \Re^{\ell_{\theta}}$ with $\ell_{\theta}$ being the length of $\theta_{0}$. Denote the conditional density of an auxiliary model by

$$
f_{t}\left(x_{t} \mid y_{t}, \beta\right), \beta \in R \subset \Re^{\ell_{\beta}} .
$$

\footnotetext{
${ }^{1}$ Some specifications in Table 1 may be different from the actual specifications used in the original references. However, they are equivalent to each other via Ito's lemma. For example, Heston (1993) adopts a square root specification for $\sigma_{t}^{2}$ which is identical to assuming $\sigma_{t}$ follows a particular OU process.
}

Further define the expected score of the auxiliary model under the structural model as

$$
m(\theta, \beta)=\int \cdots \int \frac{\partial}{\partial \beta} \ln f(x \mid y, \beta) p(x \mid y, \theta) p(y \mid \theta) d x d y .
$$

Obviously, in the context of the SV model, the integration cannot be solved analytically since neither $p(x \mid y, \theta)$ nor $p(y \mid \theta)$ has closed form. However, it is easy to simulate from an SV model so that one can approximate the integral by Monte Carlo simulations. That is

$$
m(\theta, \beta) \approx m_{N}(\theta, \beta) \equiv \frac{1}{N} \sum_{\tau=1}^{N} \frac{\partial}{\partial \beta} \ln f\left(\hat{x}_{\tau}(\theta) \mid \hat{y}_{\tau}(\theta), \beta\right),
$$

where $\left\{\hat{x}_{\tau}, \hat{y}_{\tau}\right\}$ are simulated from the structural model. The EMM estimator is a minimum chi-squared estimator which minimizes the following quadratic form,

$$
\hat{\theta}_{n}=\arg \min _{\theta \in \Theta} m_{N}^{\prime}\left(\theta, \hat{\beta}_{n}\right)\left(I_{n}\right)^{-1} m_{N}\left(\theta, \hat{\beta}_{n}\right),
$$

where $\hat{\beta}_{n}$ is a quasi maximum likelihood estimator of the auxiliary model and $I_{n}$ is an estimate of

$$
I_{0}=\lim _{n \rightarrow \infty} \operatorname{Var}\left(\frac{1}{\sqrt{n}} \sum_{t=1}^{n}\left\{\frac{\partial}{\partial \beta} \ln f_{t}\left(x_{t} \mid y_{t}, \beta^{*}\right)\right\}\right)
$$

with $\beta^{*}$ being the pseudo true value of $\beta$. Under regularity conditions, Gallant and Tauchen (1996) show that the EMM estimator is consistent and has the following asymptotic normal distribution,

$$
\sqrt{n}\left(\hat{\theta}_{n}-\theta_{0}\right) \stackrel{d}{\rightarrow} N\left(0, \frac{\partial}{\partial \theta} m\left(\theta_{0}, \beta^{*}\right)\left(I_{0}\right)^{-1} \frac{\partial}{\partial \theta^{\prime}} m\left(\theta_{0}, \beta^{*}\right)\right) .
$$

For specification testing, we have

$$
J_{n}=n m_{N}^{\prime}\left(\hat{\theta}_{n}, \hat{\beta}_{n}\right)\left(I_{n}\right)^{-1} m_{N}\left(\hat{\theta}_{n}, \hat{\beta}_{n}\right) \stackrel{d}{\rightarrow} \chi_{\ell_{\beta}-\ell_{\theta}}^{2}
$$

under the null hypothesis that the structural model is correct. When a model fails the above specification test one may wish to examine the quasi-t-ratios and/or t-ratios to look for some suggestion as to what is wrong with the structural model. Large quasi-t-ratios and t-ratios reveal the features of the data that the structural model cannot approximate.

Furthermore, Gallant and Tauchen (1996) show that if the auxiliary model nests the data generating process, EMM has the same asymptotic variance as maximum likelihood and hence is fully efficient. If the auxiliary model closely approximate the data generating process, EMM is nearly fully efficient (Gallant and Long (1997)).

To choose an auxiliary model, the seminonparametric (SNP) density proposed by Gallant and Tauchen (1989) can be used since its success has been documented in many applications. As to SNP modeling, six out of eight tuning 
parameters are to be selected, namely, $L_{u}, L_{g}, L_{r}, L_{p}, K_{z}$, and $K_{y}$. The other two parameters, $I_{z}$ and $I_{x}$, are irrelevant for univariate time series and hence are set to $0 . L_{u}$ determines the location transformation whereas $L_{g}$ and $L_{r}$ determine the scale transformation. Altogether they determine the nature of the leading term of the Hermite expansion. The other two parameters $K_{z}$ and $K_{y}$ determine the nature of the innovation. To search for a good auxiliary model, one can use the Schwarz BIC criterion to move along an upward expansion path until an adequate model is found, as outlined in Bansal et al. (1995). To preserve space we refer readers to Gallant and Tauchen (2001b) for further discussion about the role of the tuning parameters and how to design an expansion path to choose them.

\section{An Empirical Application}

In this section we consider an application using a stock index series which consist of 4044 observations on 100 times the log-first difference of the daily S\&P 500 index for the period from January 4, 1977 to December 31, 1992. The same data have been used in Gallant and Tauchen (2001a) to fit the SV model of Clark (1973). Since the return series is not mean-adjusted, to allow for a possible no-zero mean and also some dynamics in mean, we introduce an $\operatorname{AR}(1)$ structure in the mean equation. As a consequence, we fit the following two models to the data:

$$
\begin{gathered}
X_{t}=\mu_{0}+c\left(X_{t-1}-\mu_{0}\right)+\exp \left(\frac{1}{2} h_{t}\right) e_{t}, \\
h_{t}=\mu+\phi\left(h_{t-1}-\mu\right)+\sigma v_{t}
\end{gathered}
$$

and

$$
\begin{gathered}
X_{t}=\mu_{0}+c\left(X_{t-1}-\mu_{0}\right)+\left(1+\delta h_{t}\right)^{1 /(2 \delta)} e_{t}, \\
h_{t}=\mu+\phi\left(h_{t-1}-\mu\right)+\sigma v_{t} .
\end{gathered}
$$

We call them the lognormal SV model and the proposed $\mathrm{SV}$ model respectively.

The same sets of tuning parameters as in Gallant and Tauchen (2001a) are employed in the SNP model, since the identical dataset is used. We report these tuning parameters in the following order

$$
\left(L_{u}, L_{g}, L_{r}, L_{p}, K_{z}, I_{z}, K_{y}, I_{y}\right) .
$$

To ensure that the chosen SNP model is reasonable, we have compared the BIC value with those from many alternative sets of tuning parameters and find that the BIC value from the chosen SNP model is one of the smallest. The set of the tuning parameters, the corresponding $\mathrm{BIC}$ value, the leading term in the Hermite expansion, the characterization of $X_{t}$ and the number of parameters in the auxiliary model are presented in Table 2 where an $\mathrm{ARCH}$ leading term is used.
Table 2: Tuning parameters for SNP modeling, BIC, Leading Term, Characterization of $X_{t}$ and the Number of $\mathrm{Pa}$ rameters in the Auxiliary Model

\begin{tabular}{c|c|c}
\hline Data & Tuning parameters & BIC \\
\hline SP500 & $(2,0,11,1,4,0,0,0)$ & 1.32715 \\
\hline \hline Leading term & Characterization of $X_{t}$ & $\ell_{\beta}$ \\
\hline ARCH & Semiparametric ARCH & 19 \\
\hline
\end{tabular}

Since the sample size is large, we believe that the choice of leading term is not crucial as long as a form of conditional heteroskedasticity has been accommodated. In a Monte Carlo study, Andersen et al. (1999) find that the EMM efficiency approaches that of maximum likelihood for larger sample size when various forms of conditional heteroskedasticity are used as the leading term. Moreover, they find that the EMM-based inferences, such as the $t$-statistic and $J_{n}$ statistic, are robust to the choice of auxiliary model when the sample size is large.

Table 3 and Table 4 report the empirical results, where we perturb starting values when doing the optimizations. Furthermore, we simulate 101,000 observations from the SV models, of which first 1,000 observations are discarded in order to let transients die out. Table 3 reports the estimates, the numerical Wald standard errors, the $95 \%$ approximate criterion-difference confidence intervals, the value of statistic $J_{n}$, and the degrees of freedom and the p-value of $J_{n}$ for the lognormal $\mathrm{SV}$ model and for the proposed SV model. Table 4 reports the quasi-t-ratios and t-ratios from the score generator for both models.

A few results emerge from these two tables. First and most interestingly, the point estimate of $\delta$ is -0.4597 . It is significantly less than 0 and hence significantly less than 0.5 and 1 . As a consequence, one has to reject the lognormal SV model and all the other SV models in Table 1. Observing that $\delta$ is not significantly different from -0.5 , to gain some idea about our estimated results, we approximate $\delta \approx-0.5$, plug the estimates into Equation (0.12) and get the following estimated variance equation:

$$
\frac{1}{\sigma_{t}}=1.2237+0.9840\left(\frac{1}{\sigma_{t-1}}-1.2237\right)+0.057 v_{t} .
$$

This compares to the estimated variance equation in the lognormal model,

$$
\ln \sigma_{t}^{2}=-0.3425+0.9846\left(\ln \sigma_{t-1}^{2}+0.3425\right)+0.1022 v_{t} .
$$

It can be easily demonstrated that the two marginal distributions are not close to each other and hence the lognormal distribution is not a good approximation to the 
Table 3: Parameter estimates, standard errors, confidence intervals, $\chi^{2}$ criterion for S\&P500

\begin{tabular}{|c|c|c|}
\hline & Lognormal SV model & Proposed SV model \\
\hline$\mu_{0}$ & $\begin{array}{c}0.0389 \\
(0.0136) \\
{[0.0123,0.0651]}\end{array}$ & $\begin{array}{c}0.0387 \\
(0.0137) \\
{[0.0122,0.0650]}\end{array}$ \\
\hline$c$ & $\begin{array}{c}0.0880 \\
(0.0159) \\
{[0.0583,0.1179]}\end{array}$ & $\begin{array}{c}0.0875 \\
(0.0158) \\
{[0.0579,0.1177]}\end{array}$ \\
\hline$\mu$ & $\begin{array}{c}-0.3425 \\
(0.0613) \\
{[-0.4593,-0.2291]}\end{array}$ & $\begin{array}{c}-0.4474 \\
(0.0812) \\
{[-0.5066,-0.3441]}\end{array}$ \\
\hline$\phi$ & $\begin{array}{c}0.9846 \\
(0.0120) \\
{[0.9657,0.9966]}\end{array}$ & $\begin{array}{c}0.9840 \\
(0.0106) \\
{[0.9736,0.9946]}\end{array}$ \\
\hline$\sigma$ & $\begin{array}{c}0.1022 \\
(0.0456) \\
{[0.0566,0.1719]}\end{array}$ & $\begin{array}{c}0.1140 \\
(0.0405) \\
{[0.0734,0.1532]}\end{array}$ \\
\hline$\delta$ & NA & $\begin{array}{c}-0.4597 \\
(0.1807) \\
{[-0.6316,-0.2885]}\end{array}$ \\
\hline$\chi^{2}$ & 25.01 & 21.65 \\
\hline $\mathrm{df}$ & 14 & 13 \\
\hline$p-$ value & 0.034 & 0.061 \\
\hline
\end{tabular}

marginal distribution of volatility. Furthermore, theoretically speaking a distributional constraint has to be imposed on $h_{t}$ in the proposed SV models to ensure nonnegativeness of $\sigma_{t}$. In the empirical applications, however, we still adopt the assumption of exact normality. To understand how restricted this assumption is, we calculate $\operatorname{Prob}\left(\sigma_{t}<0\right)=\operatorname{Prob}\left(1 / \sigma_{t}<0\right)=0.0000065$ which is a very small value. Besides, our model is not limited to the Box-Cox power transform. Some other transformations, which do not have this restriction (such as the one in Yang (2006)), may be used.

Second, the point estimate of $\phi(0.984)$ is close to 1 and just in the stationary region. It remains almost the same in the proposed model. In fact all the estimated parameters have similar magnitude and standard errors across both models. The only exceptions are $\mu$ which decreases from -0.3425 to -0.4474 and $\sigma$ which increases from 0.1022 to 0.1140 . This is because $\mu$ and $\sigma$ are closely related to $\delta$ in the proposed model. Since the estimated $\delta$ is far away from 0 in the proposed model, this translates
Table 4: Quasi-t-ratios and t-ratios for S\&P500

\begin{tabular}{c|c|c|c|c|c}
\hline & & \multicolumn{2}{|c|}{ Lognormal SV model } & \multicolumn{2}{c}{ Proposed SV model } \\
\hline & & Quasi-t-ratio & T-ratio & Quasi-t-ratio & T-ratio \\
\hline VAR & $b_{1}$ & -0.096 & -0.334 & -0.089 & -0.302 \\
& $b_{2}$ & -1.243 & -1.249 & -1.243 & -1.259 \\
& $b_{3}$ & -0.446 & -1.540 & -0.436 & -1.571 \\
\hline \multirow{2}{*}{ ARCH } & $r_{1}$ & 0.781 & 1.391 & 0.676 & 1.471 \\
& $r_{2}$ & 0.627 & 0.909 & 0.513 & 0.896 \\
& $r_{3}$ & 0.130 & 0.224 & 0.028 & 0.051 \\
& $r_{4}$ & 1.122 & 1.228 & 1.047 & 1.160 \\
& $r_{5}$ & 1.053 & 1.498 & 0.933 & 1.399 \\
& $r_{6}$ & 0.253 & 0.312 & 0.132 & 0.179 \\
& $r_{7}$ & 0.066 & 0.088 & -0.052 & -0.071 \\
& $r_{8}$ & 1.032 & 1.521 & 0.861 & 1.489 \\
& $r_{9}$ & 0.881 & 1.040 & 0.754 & 0.945 \\
& $r_{10}$ & 0.549 & 0.588 & 0.448 & 0.494 \\
& $r_{11}$ & -0.243 & -0.280 & -0.388 & -0.500 \\
& $r_{12}$ & 0.257 & 0.270 & 0.145 & 0.159 \\
\hline \multirow{2}{*}{ SNP } & $s_{1}$ & 0.033 & 0.063 & 0.093 & 0.179 \\
& $s_{2}$ & 0.977 & 3.091 & 0.706 & 2.477 \\
& $s_{3}$ & 0.205 & 0.232 & 0.258 & 0.291 \\
& $s_{4}$ & 2.303 & 3.767 & 1.798 & 3.332 \\
\hline
\end{tabular}

to large discrepancies between the estimated $\mu$ 's and the estimated $\sigma$ 's. Third, the minimum $\chi^{2}$ criterion provides mild evidence against the lognormal specification, which is rejected at the $5 \%$ level but not at the $1 \%$ level. The evidence is consistent with the diagnostic quasi-t-ratios and t-ratios. There are large quasi-t-ratios and t-ratios on the scores corresponding to the polynomial part of the SNP score. These t-statistics indicate that $\exp \left(0.5 h_{t}\right)$ may not be the correct transformation. When the proposed SV model is fitted, the $p$-value of $J_{n}$ statistic increases by about $80 \%$. One can accept the proposed model at the $5 \%$ level. Furthermore, all the quasi-t-ratios become insignificant in the proposed model. Although some of the t-ratios on the scores corresponding to the polynomial part of the SNP score are still too large, they are clearly smaller than those in the lognormal model.

\section{References}

[1] Andersen, T. (1994). Stochastic autoregressive volatility: A framework for volatility modeling. Math. Finance 4, 75-102.

[2] Andersen, T., T. Bollerslev, F.X. Diebold, and H. Ebens (2001). The distribution of realized stock re- 
turn volatility. J. of Financial Economics 61, 43-76.

[3] Andersen, T., H. Chung and B. Sorensen (1999). Efficient method of moments estimation of a stochastic volatility model: A Monte Carlo study. J. of Econometrics $91,61-87$

[4] Bansal, R., A.R. Gallant, R. Hussey and G. Tauchen (1995) Nonparametric estimation of structural models for high-frequency currency market data. J. of Econometrics 66, 251-287.

[5] Bollerslev, T. (1986) Generalized autoregressive conditional heteroskedasticity. J. of Econometrics 31, $307-327$.

[6] Box, G.E.P. and D.R. Cox (1964). An analysis of transformations. J. Roy. Stat. Soc. Ser. B 26, 211243.

[7] Chesney, M. and L.O. Scott (1989). Pring European currency options: A comparison of the modified Black-Scholes model and a random variance model. $J$. of Financial and Quantitative Analysis 24, 267-284.

[8] Clark, P.K. (1973). A subordinated stochastic process model with finite variance for speculative price. Econometrica 41, 135-155.

[9] Engle, R.F. (1982) Autoregressive conditional heteroscedasticity with estimates of the variance of United Kingdom inflation. Econometrica 50, 9871007.

[10] Gallant, A.R. and L.R. Long (1997). Estimating stochastic differential equations efficiently by minimum chi-squared. Biometrika 84, 125-141.

[11] Gallant, A.R. and G. Tauchen (1989). Seminonparametric estimation of conditionally constrained heterogeneous processes: Asset pricing applications. Econometrica 57, 1091-1120.

[12] Gallant, A.R. and G. Tauchen (1996). Which moments to match? Econometric Theory 12, 657-681.

[13] Gallant, A.R. and G. Tauchen (2001a). EMM: A program for efficient method of moments estimation. User's Guide, Department of Economics, University of North Carolina.

[14] Gallant, A.R. and G. Tauchen (2001b). SNP: A program for nonparametric time series analysis. User's Guide, Department of Economics, University of North Carolina.

[15] Hansen, L.P. (1982). Large sample properties of generalized method of moments estimators. Econometrica 50, 1029-1054.
[16] Hentschel, L. (1995). All in the family: Nesting symmetric and asymmetric GARCH models. J. Financial Economics 39, 71-104.

[17] Heston, S.L. (1993). A closed-form solution for options with stochastic volatility, with application to bond and currency options. Rev. of Financial Stud. 6, 327-343.

[18] Higgins, M.L. and A. Bera (1992). A class of nonlinear ARCH models. International Economic Rev. 33, $137-158$.

[19] Hull, J. and A. White (1987). The pricing of options on assets with stochastic volatilities. J. of Finance $42,281-300$.

[20] Johnson, H. and D. Shanno (1987). Option pricing when the variance is changing. J. of Financial and Quantitative Analysis 22, 143-152.

[21] Kim, S., N. Shephard, and S. Chib (1998). Stochastic volatility: Likelihood inference and comparison with ARCH models. Rev. of Economic Stud. 65, 361-393.

[22] Melino, A. and S.M. Turnbull (1990). Pricing foreign currency options with stochastic volatility. J. of Econometrics 45, 239-265.

[23] Scott, L.O. (1987). Option pricing when the variance changes randomly: Theory, estimation and an application. J. of Financial and Quantitative Analysis 22, 419-439.

[24] Stein, E.M. and J.C. Stein (1991). Stock price distributions with stochastic volatility: An analytical approach. Rev. of Financial Stud. 4, 727-752.

[25] Wiggins, J.B. (1987) Option values under stochastic volatility: Theory and empirical estimate. J. of Financial Economics 19, 351-372.

[26] Yang, Z. (2006) A Modified Family of Power Transformations. Economics Letters 92, 14-19.

[27] Yu, J. (2002) Forecasting volatility in the New Zealand stock market. Applied Financial Economics 12, 193-202. 